Research Article

\title{
Some Identities Involving Derangement Polynomials and Numbers and Moments of Gamma Random Variables
}

\author{
Lee-Chae Jang, ${ }^{1}$ Dae San Kim, ${ }^{2}$ Taekyun Kim $\mathbb{D D}^{3}{ }^{3}$ and Hyunseok Lee ${ }^{3}$ \\ ${ }^{1}$ Graduate School of Education, Konkuk University, Seoul 143-701, Republic of Korea \\ ${ }^{2}$ Department of Mathematics, Sogang University, Seoul 121-742, Republic of Korea \\ ${ }^{3}$ Department of Mathematics, Kwangwoon University, Seoul 139-701, Republic of Korea
}

Correspondence should be addressed to Taekyun Kim; kwangwoonmath@hanmail.net

Received 5 November 2020; Revised 7 December 2020; Accepted 12 December 2020; Published 16 December 2020

Academic Editor: Ioan Rasa

Copyright ( 92020 Lee-Chae Jang et al. This is an open access article distributed under the Creative Commons Attribution License, which permits unrestricted use, distribution, and reproduction in any medium, provided the original work is properly cited.

The problem of counting derangements was initiated by Pierre Rémond de Montmort in 1708. A derangement is a permutation that has no fixed points, and the derangement number $D_{n}$ is the number of fixed point free permutations on an $n$ element set. Furthermore, the derangement polynomials are natural extensions of the derangement numbers. In this paper, we study the derangement polynomials and numbers, their connections with cosine-derangement polynomials and sine-derangement polynomials, and their applications to moments of some variants of gamma random variables.

\section{Introduction and Preliminaries}

The problem of counting derangements was initiated by Pierre Rémond de Montmort in 1708 (see [1, 2]). A derangement is a permutation of the elements of a set, such that no element appears in its original position. In other words, a derangement is a permutation that has no fixed points. The derangement number $D_{n}$ is the number of fixed point free permutations on an $n(n \geq 1)$ element set.

The aim of this paper is to study derangement polynomials and numbers, their connections with cosinederangement polynomials and sine-derangement polynomials, and their applications to moments of some variants of gamma random variables. Here, the derangement polynomials $D_{n}(x)$ are natural extensions of the derangement numbers.

The outline of our main results is as follows. We show a recurrence relation for derangement polynomials. Then, we derive identities involving derangement polynomials, Bell polynomials, and Stirling numbers of both kinds. In addition, we also have an identity relating Bell polynomials, derangement polynomials, and Euler numbers. Next, we introduce the two variable polynomials, namely, cosine-derangement polynomials $D_{n}^{(c)}(x, y)$ and sine-derangement polynomials $D_{n}^{(s)}(x, y)$, in a natural manner by means of derangement polynomials. We obtain, among other things, their explicit expressions and recurrence relations. Lastly, in the final section, we show that if $X$ is the gamma random variable with parameters 1,1 , then $D_{n}(p), D_{n}^{(c)}(p, q), D_{n}^{(s)}(p, q)$ are given by the "moments" of some variants of $X$.

In the rest of this section, we recall the derangement numbers, especially their explicit expressions, generating function, and recurrence relations. Also, we give the derangement polynomials and give their explicit expressions. Then, we recall the gamma random variable with parameters $\alpha, \lambda$ along with their moments and the Bell polynomials. Finally, we give the definitions of the Stirling numbers of the first and second kinds.

As before, let $D_{n}$ denote the derangement number for $n$ $\geq 1$, and let $D_{0}=1$. Then, the first few derangement numbers $D_{n}(n \geq 0)$ are $1,0,1,2,9,44,265,1854,14833,133496$, $1334961, \cdots$. For $n \geq 0$, the derangement numbers are given by [3-5]

$$
\begin{aligned}
D_{n} & =n !-\left(\begin{array}{l}
n \\
1
\end{array}\right)(n-1) !+\left(\begin{array}{l}
n \\
2
\end{array}\right)(n-2) !-\left(\begin{array}{l}
n \\
3
\end{array}\right)(n-3) !+\cdots+(-1)^{n}\left(\begin{array}{l}
n \\
n
\end{array}\right) 0 ! \\
& =\sum_{k=0}^{n}\left(\begin{array}{l}
n \\
k
\end{array}\right)(n-k) !(-1)^{k}=n ! \sum_{k=0}^{n} \frac{(-1)^{k}}{k !} .
\end{aligned}
$$


From (1), we note that $[1-4,6,7]$

$$
\sum_{n=0}^{\infty} D_{n} \frac{t^{n}}{n !}=\frac{1}{1-t} e^{-t}
$$

By (2), we get

$$
e^{-t}=(1-t) \sum_{n=0}^{\infty} D_{n} \frac{t^{n}}{n !}=1+\sum_{n=1}^{\infty}\left(D_{n}-n D_{n-1}\right) \frac{t^{n}}{n !}
$$

From (3), we can easily derive the following recurrence relation $[5,8-11]$ :

$$
(-1)^{n}=D_{n}-n D_{n-1},(n \geq 1) .
$$

Now, we consider the derangement polynomials which are given by [10]

$$
\frac{e^{-t}}{1-t} e^{x t}=\sum_{n=0}^{\infty} D_{n}(x) \frac{t^{n}}{n !}
$$

From (5), we have

$$
\sum_{n=0}^{\infty} D_{n}(x) \frac{t^{n}}{n !}=\frac{1}{1-t} e^{-t} e^{x t}=\sum_{n=0}^{\infty}\left(\sum_{l=0}^{n}\left(\begin{array}{l}
n \\
l
\end{array}\right) D_{l} x^{n-l}\right) \frac{t^{n}}{n !}
$$
[10]

By comparing the coefficients on both sides of (6), we get

$$
D_{n}(x)=\sum_{l=0}^{n}\left(\begin{array}{l}
n \\
l
\end{array}\right) D_{l} x^{n-l},(n \geq 0) .
$$

On the other hand,

$$
\begin{aligned}
\frac{e^{-t}}{1-t} e^{x t} & =\frac{1}{1-t} e^{(x-1) t}=\sum_{l=0}^{\infty} t^{l} \sum_{m=0}^{\infty}(x-1)^{m} \frac{t^{m}}{m !} \\
& =\sum_{n=0}^{\infty}\left(n ! \sum_{m=0}^{n} \frac{(x-1)^{m}}{m !}\right) \frac{t^{n}}{n !} .
\end{aligned}
$$

From (6), (7), and (8), we have

$$
D_{n}(x)=n ! \sum_{m=0}^{n} \frac{(x-1)^{m}}{m !}=\sum_{l=0}^{n}\left(\begin{array}{l}
n \\
l
\end{array}\right) D_{l} x^{n-l},(n \geq 0) .
$$

A continuous random variable $X$ whose density function is given by [12-14]

$$
f(x)= \begin{cases}\lambda e^{-\lambda x} \frac{(\lambda x)^{\alpha-1}}{\Gamma(\alpha)}, & \text { if } x \geq 0, \\ 0, & \text { if } x<0\end{cases}
$$

for some $\lambda>0$ and $\alpha>0$ is said to be the gamma random variable with parameter $\alpha, \lambda$ which is denoted by $X \sim \Gamma(\alpha, \lambda)$.

For $X \sim \Gamma(\alpha, \lambda)$, the $n$-th moment of $X$ is given by

$$
\begin{aligned}
E\left[X^{n}\right] & =\frac{\lambda}{\Gamma(\alpha)} \int_{0}^{\infty} x^{n} e^{-\lambda x}(\lambda x)^{\alpha-1} d x \\
& =\frac{1}{\lambda^{n} \Gamma(\alpha)} \int_{0}^{\infty} t^{n+\alpha-1} e^{-t} d t \\
& =\frac{\Gamma(\alpha+n)}{\lambda^{n} \Gamma(\alpha)}=\frac{(\alpha+n) \cdots(\alpha+1) \alpha}{\lambda^{n}} .
\end{aligned}
$$
[15]

It is well known that the Bell polynomials are defined by

$$
e^{x\left(e^{t}-1\right)}=\sum_{n=0}^{\infty} \operatorname{Bel}_{n}(x) \frac{t^{n}}{n !} .
$$

When $x=1, \operatorname{Bel}_{n}=\operatorname{Bel}_{n}(1)(n \geq 0)$ are called the Bell numbers

The Stirling numbers of the first kind are defined as [16, 17]

$$
(x)_{n}=\sum_{l=0}^{n} S_{1}(n, l) x^{l},(n \geq 0)
$$

where $(x)_{0}=1,(x)_{n}=x(x-1) \cdots(x-n+1)(n \geq 1)$.

As an inversion formula of (13), the Stirling numbers of the second kind are defined by [16-18]

$$
x^{n}=\sum_{l=0}^{n} S_{2}(n, l)(x)_{l}(n \geq 0) .
$$

\section{Derangement Polynomials and Numbers}

From (5), we have

$e^{(x-1) t}=\left(\sum_{n=0}^{\infty} D_{n}(x) \frac{t^{n}}{n !}\right)(1-t)=1+\sum_{n=1}^{\infty}\left(D_{n}(x)-n D_{n-1}(x)\right) \frac{t^{n}}{n !}$.

On the other hand,

$$
e^{(x-1) t}=\sum_{n=0}^{\infty} \frac{(x-1)^{n}}{n !} t^{n}=1+\sum_{n=1}^{\infty} \frac{(x-1)^{n}}{n !} t^{n} .
$$

Therefore, by (15) and (16), we obtain the following lemma.

Lemma 1. For $n \geq 1$, we have

$$
D_{n}(x)-n D_{n-1}(x)=(x-1)^{n} .
$$


Replacing $t$ by $1-e^{t}$ in (5), we get

$$
\begin{aligned}
e^{(1-x)\left(e^{t}-1\right)} & =e^{t} \sum_{l=0}^{\infty} D_{l}(x) \frac{1}{l !}\left(1-e^{t}\right)^{l} \\
& =\sum_{m=0}^{\infty} \frac{t^{m}}{m !} \sum_{l=0}^{\infty}(-1)^{l} D_{l}(x) \sum_{j=l}^{\infty} S_{2}(j, l) \frac{t^{j}}{j !} \\
& =\sum_{m=0}^{\infty} \frac{t^{m}}{m !} \sum_{j=0}^{\infty}\left(\sum_{l=0}^{j}(-1)^{l} D_{l}(x) S_{2}(j, l) \frac{t^{j}}{j !}\right. \\
& =\sum_{n=0}^{\infty}\left(\sum_{j=0}^{n} \sum_{l=0}^{j}\left(\begin{array}{l}
n \\
j
\end{array}\right)(-1)^{l} D_{l}(x) S_{2}(j, l)\right) \frac{t^{n}}{n !} .
\end{aligned}
$$

From (18), we have

$$
\operatorname{Bel}_{n}(1-x)=\sum_{j=0}^{n} \sum_{l=0}^{j}\left(\begin{array}{l}
n \\
j
\end{array}\right)(-1)^{l} D_{l}(x) S_{2}(j, l),(n \geq 0) .
$$

It is easy to show that

$$
\begin{aligned}
\frac{1}{e^{t}} e^{(1-x)\left(e^{t}-1\right)} & =\sum_{l=0}^{\infty} \frac{(-1)^{l}}{l !} t^{l} \sum_{m=0}^{\infty} \operatorname{Bel}_{m}(1-x) \frac{t^{m}}{m !} \\
& =\sum_{n=0}^{\infty}\left(\sum_{m=0}^{n}\left(\begin{array}{l}
n \\
m
\end{array}\right) \operatorname{Bel}_{m}(1-x)(-1)^{n-m}\right) \frac{t^{n}}{n !}
\end{aligned}
$$

Replacing $t$ by $\log (1-t)$ in (20), we get

$$
\begin{aligned}
\frac{1}{1-t} e^{-t} e^{x t} & =\sum_{l=0}^{\infty} \sum_{m=0}^{l}\left(\begin{array}{l}
l \\
m
\end{array}\right) \operatorname{Bel}_{m}(1-x)(-1)^{l-m} \frac{1}{l !}(\log (1-t))^{l} \\
& =\sum_{l=0}^{\infty} \sum_{m=0}^{l}\left(\begin{array}{l}
l \\
m
\end{array}\right) \operatorname{Bel}_{m}(1-x)(-1)^{l-m} \sum_{n=l}^{\infty}(-1)^{n} S_{1}(n, l) \frac{t^{n}}{n !} \\
& =\sum_{n=0}^{\infty}\left(\sum_{l=0}^{n} \sum_{m=0}^{l}\left(\begin{array}{l}
l \\
m
\end{array}\right) \operatorname{Bel}_{m}(1-x)(-1)^{n-l-m} S_{1}(n, l)\right) \frac{t^{n}}{n !} .
\end{aligned}
$$

From (5) and (21), we have

$$
D_{n}(x)=\sum_{l=0}^{n} \sum_{m=0}^{l}\left(\begin{array}{l}
l \\
m
\end{array}\right) \operatorname{Bel}_{m}(1-x)(-1)^{n-m-l} S_{1}(n, l),(n \geq 0) .
$$

Therefore, by (19) and (22), we obtain the following theorem.
Theorem 2. For $n \geq 0$, we have

$$
\begin{gathered}
\operatorname{Bel}_{n}(1-x)=\sum_{j=0}^{n} \sum_{l=0}^{j}\left(\begin{array}{l}
n \\
j
\end{array}\right)(-1)^{l} D_{l}(x) S_{2}(j, l), \\
D_{n}(x)=\sum_{l=0}^{n} \sum_{m=0}^{l}\left(\begin{array}{c}
l \\
m
\end{array}\right) B e l_{m}(1-x)(-1)^{n-m-l} S_{l}(n, l) .
\end{gathered}
$$

Corollary 3. For $n \geq 0$, we have

$$
B e l_{n}=\sum_{j=0}^{n} \sum_{l=0}^{j}\left(\begin{array}{l}
n \\
j
\end{array}\right)(-1)^{l} D_{l} S_{2}(j, l)
$$

$$
D_{n}=\sum_{l=0}^{n} \sum_{m=0}^{l}\left(\begin{array}{l}
l \\
m
\end{array}\right) \operatorname{Bel}_{m}(-1)^{n-m-l} S_{1}(n, l) \text {. }
$$

Replacing $t$ by $-e^{t}$ in (5), we get

$$
\begin{aligned}
\frac{1}{e^{t}+1} e^{(1-x) e^{t}} & =\sum_{m=0}^{\infty} D_{m}(x) \frac{(-1)^{m}}{m !} e^{m t} \\
& =\sum_{m=0}^{\infty} \frac{D_{m}(x)(-1)^{m}}{m !} \sum_{n=0}^{\infty} m^{n} \frac{t^{n}}{n !} \\
& =\sum_{n=0}^{\infty}\left(\sum_{m=0}^{\infty} \frac{(-1)^{m} D_{m}(x)}{m !} m^{n}\right) \frac{t^{n}}{n !} .
\end{aligned}
$$

On the other hand, we have

$$
\begin{aligned}
\frac{1}{e^{t}+1} e^{(1-x) e^{t}} & =\frac{e^{1-x}}{2} \frac{2}{e^{t}+1} e^{(1-x)\left(e^{t}-1\right)} \\
& =\frac{e^{1-x}}{2} \sum_{l=0}^{\infty} E_{l} \frac{t^{l}}{l !} \sum_{m=0}^{\infty} \operatorname{Bel}_{m}(1-x) \frac{t^{m}}{m !} \\
& =\frac{e^{1-x}}{2} \sum_{n=0}^{\infty}\left(\sum_{m=0}^{n} \operatorname{Bel}_{m}(1-x) E_{n-m}\left(\begin{array}{c}
n \\
m
\end{array}\right)\right) \frac{t^{n}}{n !},
\end{aligned}
$$

where $E_{n}$ are the ordinary Euler numbers.

Therefore, by (25) and (26), we obtain the following theorem.

Theorem 4. For $n \geq 0$, we have

$$
\sum_{m=0}^{n} \operatorname{Bel}_{m}(1-x) E_{n-m}\left(\begin{array}{l}
n \\
m
\end{array}\right)=2 e^{x-1} \sum_{m=0}^{\infty}(-1)^{m} \frac{D_{m}(x)}{m !} m^{n} .
$$


Now, we observe that

$$
\begin{aligned}
& \left(\frac{1}{1-t}\right)^{r}=\left(\frac{1}{1-t}\right)^{r} e^{-r t} e^{r t}=\left(\frac{1}{1-t} e^{-t}\right)^{r-1} \frac{e^{-t}}{1-t} e^{r t} \\
& =\sum_{k=0}^{\infty} \sum_{l_{1}+\cdots+l_{r-1}=k}\left(\begin{array}{c}
k \\
l_{1}, \cdots, l_{r-1}
\end{array}\right) D_{l_{1}} D_{l_{2}} \cdots D_{l_{r-1}} \\
& \cdot \frac{t^{k}}{k !} \sum_{m=0}^{\infty} D_{m}(r) \frac{t^{m}}{m !} \\
& =\sum_{n=0}^{\infty}\left(\sum_{k=0}^{n} \sum_{l_{1}+\cdots+l_{r-1}=k}\left(\begin{array}{c}
k \\
l_{1}, \cdots, l_{r-1}
\end{array}\right)\right. \\
& \left.\cdot\left(\begin{array}{l}
n \\
k
\end{array}\right) D_{l_{1}} D_{l_{2}} \cdots D_{l_{r-1}} D_{n-k}(r)\right) \frac{t^{n}}{n !},
\end{aligned}
$$

where $r$ is a positive integer.

On the other hand,

$$
\left(\frac{1}{1-t}\right)^{r}=\sum_{n=0}^{\infty}\left(\begin{array}{c}
-r \\
n
\end{array}\right)(-1)^{n} t^{n}=\sum_{n=0}^{\infty} n !\left(\begin{array}{c}
r+n-1 \\
n
\end{array}\right) \frac{t^{n}}{n !} .
$$

Therefore, by (28) and (29), we obtain the following proposition.

Proposition 5. For $r \in \mathbb{N}$, we have

$$
\left(\begin{array}{c}
r+n-1 \\
n
\end{array}\right)=\frac{1}{n !} \sum_{k=0}^{n} \sum_{l_{1}+\cdots+l_{r-1}=k}\left(\begin{array}{c}
k \\
l_{1}, \cdots, l_{r-1}
\end{array}\right)\left(\begin{array}{l}
n \\
k
\end{array}\right) D_{l_{1}} \cdots D_{l_{r-1}} D_{n-k}(r) .
$$

It is well known that $[16,18,19]$

$$
e^{i x}=\cos x+i \sin x, \quad i=\sqrt{-1} .
$$

From (5), we note that

$$
\begin{gathered}
\frac{e^{-t}}{1-t} e^{(x+i y) t}=\sum_{n=0}^{\infty} D_{n}(x+i y) \frac{t^{n}}{n !},(x, y \in \mathbb{R}), \\
\frac{e^{-t}}{1-t} e^{(x-i y) t}=\sum_{n=0}^{\infty} D_{n}(x-i y) \frac{t^{n}}{n !} .
\end{gathered}
$$

By (9), (32), and (33), we get

$$
\begin{gathered}
D_{n}(x+i y)=n ! \sum_{m=0}^{n} \frac{(x-1+i y)^{m}}{m !}, \\
D_{n}(x-i y)=n ! \sum_{m=0}^{n} \frac{(x-1-i y)^{m}}{m !},(n \geq 0) .
\end{gathered}
$$

From (34) and (35), we can derive the following equations:

$$
\begin{aligned}
& \frac{e^{-t}}{1-t} e^{x t} \cos (y t)=\sum_{n=0}^{\infty}\left(\frac{D_{n}(x+i y)+D_{n}(x-i y)}{2}\right) \frac{t^{n}}{n !} \\
& \frac{e^{-t}}{1-t} e^{x t} \sin (y t)=\sum_{n=0}^{\infty}\left(\frac{D_{n}(x+i y)-D_{n}(x-i y)}{2 i}\right) \frac{t^{n}}{n !}
\end{aligned}
$$

We define cosine-derangement polynomials and sinederangement polynomials, respectively, by

$$
\begin{gathered}
\frac{e^{-t}}{1-t} e^{x t} \cos y t=\sum_{n=0}^{\infty} D_{n}^{(c)}(x, y) \frac{t^{n}}{n !} \\
\frac{e^{-t}}{1-t} e^{x t} \sin y t=\sum_{n=0}^{\infty} D_{n}^{(s)}(x, y) \frac{t^{n}}{n !}
\end{gathered}
$$

Thus, we have

$$
\begin{gathered}
D_{n}^{(c)}(x, y)=\frac{D_{n}(x+i y)+D_{n}(x-i y)}{2}, \\
D_{n}^{(s)}(x, y)=\frac{D_{n}(x+i y)-D_{n}(x-i y)}{2 i},(n \geq 0) .
\end{gathered}
$$

Therefore, we obtain the following theorem.

Theorem 6. For $n \geq 0$, we have

$$
\begin{aligned}
& D_{n}^{(c)}(x, y)=\frac{n !}{2} \sum_{m=0}^{n} \frac{1}{m !}\left((x-1+i y)^{m}+(x-1-i y)^{m}\right), \\
& D_{n}^{(s)}(x, y)=\frac{n !}{2 i} \sum_{m=0}^{n} \frac{1}{m !}\left((x-1+i y)^{m}-(x-1-i y)^{m}\right) .
\end{aligned}
$$

Before proceeding further, we recall that

$$
\cos y t=\sum_{n=0}^{\infty} \frac{(-1)^{n}}{(2 n) !} y^{2 n} t^{2 n}
$$

From (38)and (42), we note that

$$
\begin{aligned}
\sum_{n=0}^{\infty} D_{n}^{(c)}(x, y) \frac{t^{n}}{n !} & =\frac{e^{-t}}{1-t} e^{x t} \cos (y t) \\
& =\sum_{l=0}^{\infty} \frac{D_{l}}{l !} t^{l} \sum_{k=0}^{\infty} \sum_{m=0}^{[k / 2]}\left(\begin{array}{c}
k \\
2 m
\end{array}\right)(-1)^{m} y^{2 m} x^{k-2 m} \frac{t^{k}}{k !} \\
& =\sum_{n=0}^{\infty}\left(\sum_{k=0}^{n}\left(\begin{array}{l}
n \\
k
\end{array}\right) D_{n-k} \sum_{m=0}^{[k / 2]}\left(\begin{array}{c}
k \\
2 m
\end{array}\right)(-1)^{m} y^{2 m} x^{k-2 m}\right) \frac{t^{n}}{n !} .
\end{aligned}
$$

Therefore, by comparing the coefficients on both sides of (43), we obtain the following theorem. 
Theorem 7. For $n \geq 0$, we have

$$
D_{n}^{(c)}(x, y)=\sum_{m=0}^{[n / 2]} \sum_{k=2 m}^{n}\left(\begin{array}{l}
n \\
k
\end{array}\right)\left(\begin{array}{c}
k \\
2 m
\end{array}\right) D_{n-k}(-1)^{m} y^{2 m} x^{k-2 m} .
$$

Corollary 8. For $n \geq 0$, we have

$$
\begin{aligned}
\frac{n !}{2} & \sum_{m=0}^{n} \frac{1}{m !}\left((x-1+i y)^{m}+(x-1-i y)^{m}\right) \\
= & \sum_{m=0}^{[n / 2]} \sum_{k=2 m}^{n}\left(\begin{array}{l}
n \\
k
\end{array}\right)\left(\begin{array}{c}
k \\
2 m
\end{array}\right) D_{n-k}(-1)^{m} y^{2 m} x^{k-2 m} .
\end{aligned}
$$

By (38), we get

$$
\begin{aligned}
e^{(x-1) t} \cos y t & =(1-t) \sum_{n=0}^{\infty} D_{n}^{(c)}(x, y) \frac{t^{n}}{n !} \\
& =1+\sum_{n=1}^{\infty}\left(D_{n}^{(c)}(x, y)-n D_{n-1}^{(c)}(x, y)\right) \frac{t^{n}}{n !} .
\end{aligned}
$$

Thus, we have

$$
\begin{aligned}
\cos y t= & e^{(1-x) t}+e^{(1-x) t} \sum_{m=1}^{\infty}\left(D_{m}^{(c)}(x, y)-m D_{m-1}^{(c)}(x, y)\right) \frac{t^{m}}{m !} \\
= & \sum_{n=0}^{\infty}(1-x)^{n} \frac{t^{n}}{n !}+\sum_{l=0}^{\infty}(1-x)^{l} \frac{t^{l}}{l !} \sum_{m=1}^{\infty} \\
& \cdot\left(D_{m}^{(c)}(x, y)-m D_{m-1}^{(c)}(x, y)\right) \frac{t^{m}}{m !} \\
= & 1+\sum_{n=1}^{\infty}\left((1-x)^{n}+\sum_{m=1}^{n}\left(\begin{array}{l}
n \\
m
\end{array}\right)(1-x)^{n-m}\right. \\
& \left.\cdot\left(D_{m}^{(c)}(x, y)-m D_{m-1}^{(c)}(x, y)\right)\right) \frac{t^{n}}{n !} .
\end{aligned}
$$

Therefore, by (47) and (42), we obtain the following theorem.

Theorem 9. For $k \in \mathbb{N}$, we have

$$
\begin{aligned}
& (1-x)^{n}+\sum_{m=1}^{n}\left(\begin{array}{c}
n \\
m
\end{array}\right)(1-x)^{n-m}\left(D_{m}^{(c)}(x, y)-m D_{m-1}^{(c)}(x, y)\right) \\
& \quad= \begin{cases}(-1)^{k} y^{2 k}, & \text { if } n=2 k, \\
0, & \text { if } n=2 k-1 .\end{cases}
\end{aligned}
$$

By (38), we get

$$
\begin{aligned}
e^{(x-1) t} \cos y t & =\sum_{n=0}^{\infty} D_{n}^{(c)}(x, y) \frac{t^{n}}{n !}(1-t) \\
& =\sum_{n=1}^{\infty}\left(D_{n}^{(c)}(x, y)-n D_{n-1}^{(c)}(x, y)\right) \frac{t^{n}}{n !}+1 .
\end{aligned}
$$

On the other hand,

$$
\begin{aligned}
e^{(x-1) t} \cos y t & =\sum_{l=0}^{\infty}(x-1)^{l} \frac{t^{l}}{l !} \sum_{m=0}^{\infty} y^{2 m}(-1)^{m} \frac{t^{2 m}}{(2 m) !} \\
& =1+\sum_{n=1}^{\infty}\left(\sum_{m=0}^{[n / 2]}\left(\begin{array}{c}
n \\
2 m
\end{array}\right)(-1)^{m}(x-1)^{n-2 m} y^{2 m}\right) \frac{t^{n}}{n !} .
\end{aligned}
$$

Therefore, by (49) and (50), we obtain the following theorem.

Theorem 10. For $n \geq 1$, we have

$$
D_{n}^{(c)}(x, y)-n D_{n-1}^{(c)}(x, y)=\sum_{m=0}^{[n / 2]}\left(\begin{array}{c}
n \\
2 m
\end{array}\right)(-1)^{m}(x-1)^{n-2 m} y^{2 m}
$$

It is not difficult to show that

$$
\sum_{n=0}^{\infty} D_{n}^{(c)}(x+r, y) \frac{t^{n}}{n !}=\sum_{n=0}^{\infty}\left(\sum_{l=0}^{n}\left(\begin{array}{l}
n \\
l
\end{array}\right) D_{l}^{(c)}(x, y) r^{n-l}\right) \frac{t^{n}}{n !},
$$

where $r$ is a positive integer.

By comparing the coefficients on both sides of (47), we get

$$
D_{n}^{(c)}(x+r, y)=\sum_{l=0}^{n}\left(\begin{array}{l}
n \\
l
\end{array}\right) D_{l}^{(c)}(x, y) r^{n-l} .
$$

Now, we observe that

$$
\begin{aligned}
\sum_{n=1}^{\infty} \frac{\partial}{\partial x} D_{n}^{(c)}(x, y) \frac{t^{n}}{n !} & =\frac{\partial}{\partial x}\left(\frac{e^{-t}}{1-t} e^{x t} \cos y t\right) \\
& =t \frac{e^{-t}}{1-t} e^{x t} \cos y t=t \sum_{n=0}^{\infty} D_{n}^{(c)}(x, y) \frac{t^{n}}{n !} \\
& =\sum_{n=1}^{\infty} n D_{n-1}^{(c)}(x, y) \frac{t^{n}}{n !} .
\end{aligned}
$$


Form (54), we note that

$D_{0}^{(c)}(x, y)=1, \frac{\partial}{\partial x} D_{n}^{(c)}(x, y)=n D_{n-1}^{(c)}(x, y),(n \geq 1)$

Therefore, we obtain the following theorem.

Theorem 11. For $n \geq 0$, we have

$$
D_{0}^{(c)}(x, y)=1, \frac{\partial}{\partial x} D_{n}^{(c)}(x, y)=n D_{n-1}^{(c)}(x, y),(n \geq 1)
$$

In particular,

$$
\frac{d}{d x} D_{n}(x)=\frac{\partial}{\partial x} D_{n}^{(c)}(x, 0)=n D_{n-1}^{(c)}(x, 0)=n D_{n-1}^{(c)}(x),(n \geq 1)
$$

Corollary 12. $D_{n}^{(c)}(x, y)$ as a polynomial in $x$, for each fixed $y$, and $D_{n}(x)$ are Appell sequences.

Before proceeding further, we recall that

$$
\sin y t=\sum_{n=1}^{\infty} \frac{(-1)^{n-1}}{(2 n-1) !} y^{2 n-1} t^{2 n-1}
$$

From (39)and (58), we note that

$$
\begin{aligned}
& \sum_{n=0}^{\infty} D_{n}^{(s)}(x, y) \frac{t^{n}}{n !}=\frac{1}{1-t} e^{-t} e^{x t} \sin y t \\
& =\sum_{k=0}^{\infty} \frac{D_{k}}{k !} t^{k} \sum_{j=1}^{\infty} \sum_{m=0}^{[(j-1) / 2]} \\
& \left(\begin{array}{c}
j \\
2 m+1
\end{array}\right) x^{j-2 m-1} y^{2 m+1} \frac{t^{j}}{j !} \\
& =\sum_{n=1}^{\infty}\left(\sum_{j=1}^{n} \sum_{m=0}^{[(j-1) / 2]}\left(\begin{array}{c}
j \\
2 m+1
\end{array}\right)\right. \\
& \left.\left(\begin{array}{l}
n \\
j
\end{array}\right) x^{j-2 m-1} y^{2 m+1} D_{n-j}\right) \frac{t^{n}}{n !} \text {. }
\end{aligned}
$$

Therefore, by (59), we obtain the following theorem.

Theorem 13. For $n \geq 0$, we have

$$
\begin{aligned}
D_{0}^{(s)}(x, y) & =0, D_{n}^{(s)}(x, y) \\
& =\sum_{j=1}^{n} \sum_{m=0}^{[(j-1) / 2]}\left(\begin{array}{c}
j \\
2 m+1
\end{array}\right)\left(\begin{array}{l}
n \\
j
\end{array}\right) x^{j-2 m-1} y^{2 m+1} D_{n-j} .
\end{aligned}
$$

By (35) and (37) and Theorem 13, we obtain the following corollary.
Corollary 14. For $n \geq 1$, we have

$$
\begin{aligned}
\frac{D_{n}(x+i y)-D_{n}(x-i y)}{2 i}= & \sum_{j=1}^{n} \sum_{m=1}^{[(j-1) / 2]}\left(\begin{array}{c}
j \\
2 m+1
\end{array}\right) \\
& \cdot\left(\begin{array}{c}
n \\
j
\end{array}\right) x^{j-2 m-1} y^{2 m+1} D_{n-j} .
\end{aligned}
$$

By (59), we see that

$$
\begin{aligned}
\sin y t= & e^{(1-x) t} \sum_{k=1}^{\infty}\left(D_{k}^{(s)}(x, y)-k D_{k-1}^{(s)}(x, y)\right) \frac{t^{k}}{k !} \\
= & \sum_{m=0}^{\infty}(1-x)^{m} \frac{t^{m}}{m !} \sum_{k=1}^{\infty}\left(D_{k}^{(s)}(x, y)-k D_{k-1}^{(s)}(x, y)\right) \frac{t^{k}}{k !} \\
= & \sum_{n=1}^{\infty}\left(\sum_{k=1}^{n}\left(\begin{array}{l}
n \\
k
\end{array}\right)\right. \\
& \left.\cdot\left(D_{k}^{(s)}(x, y)-k D_{k-1}^{(s)}(x, y)\right)(1-x)^{n-k}\right) \frac{t^{n}}{n !} .
\end{aligned}
$$

Therefore, by (62) and (58), we obtain the following theorem.

Theorem 15. For $m \in \mathbb{N}$, we have

$$
\begin{aligned}
& \sum_{k=1}^{n}\left(\begin{array}{l}
n \\
k
\end{array}\right)\left(D_{k}^{(s)}(x, y)-k D_{k-1}^{(s)}(x, y)\right)(1-x)^{n-k} \\
& \quad= \begin{cases}(-1)^{m-1} y^{2 m-1}, & \text { if } n=2 m-1, \\
0, & \text { if } n=2 m .\end{cases}
\end{aligned}
$$

It is easy to show that $(\partial / \partial x) D_{n}^{(s)}(x, y)=n D_{n-1}^{(s)}(x, y)$. However, $D_{n}^{(s)}(x, y)$ is not an Appell sequence, since $D_{0}^{(s)}(x$, $y)=0$.

We observe that

$$
\begin{aligned}
\sum_{n=0}^{\infty} D_{n}^{(s)}(x, y) \frac{t^{n}}{n !}= & \frac{e^{-t}}{1-t} e^{x t} \sin y t \\
= & \sum_{l=0}^{\infty} D_{l}(x) \frac{t^{l}}{l !} \sum_{m=0}^{\infty}(-1)^{m} y^{2 m+1} \frac{t^{2 m+1}}{(2 m+1) !} \\
= & \sum_{n=1}^{\infty}\left(\sum_{m=0}^{[(n-1) / 2]}\left(\begin{array}{c}
n \\
2 m+1
\end{array}\right)\right. \\
& \left.\cdot(-1)^{m} y^{2 m+1} D_{n-2 m-1}(x)\right) \frac{t^{n}}{n !} .
\end{aligned}
$$

Comparing the coefficients on both sides of (64), we have the following theorem. 
Theorem 16. For $n \geq 1$, we have

$$
D_{n}^{(s)}(x, y)=\sum_{m=0}^{[(n-1) / 2]}\left(\begin{array}{c}
n \\
2 m+1
\end{array}\right)(-1)^{m} y^{2 m+1} D_{n-2 m-1}(x) \text {. }
$$

For $r \in \mathbb{N}$, we have

$$
\begin{aligned}
\sum_{n=0}^{\infty} D_{n}^{(s)}(x+r, y) & =\frac{e^{-t}}{1-t} e^{(x+r) t} \sin y t=\frac{e^{-t}}{1-t} e^{x t} \sin y t e^{r t} \\
& =\sum_{l=0}^{\infty} D_{l}^{(s)}(x, y) \frac{t^{l}}{l !} \sum_{m=0}^{\infty} r^{m} \frac{t^{m}}{m !} \\
& =\sum_{n=0}^{\infty}\left(\sum_{l=0}^{n}\left(\begin{array}{l}
n \\
l
\end{array}\right) D_{l}^{(s)}(x, y) r^{n-l}\right) \frac{t^{n}}{n !} .
\end{aligned}
$$

Thus, we obtain

$$
D_{n}^{(s)}(x+r, y)=\sum_{l=0}^{n}\left(\begin{array}{l}
n \\
l
\end{array}\right) D_{l}^{(s)}(x, y) r^{n-l},(n \geq 0) .
$$

\section{Further Remarks}

As applications, we want to show that if $X$ is the gamma random variable with parameters 1,1 , then $D_{n}(p), D_{n}^{(c)}(\mathrm{p}, q)$, $D_{n}^{(s)}(p, q)$ are given by the "moments" of some variants of $X$ . We let the reader refer to the papers [20-22] for some recent papers related to this section.

Let $X$ be a gamma random variable with parameters 1,1 which is denoted by $X \sim \Gamma(1,1)$. Then, we observe that

$$
E\left[e^{(X-1+p) t}\right]=\int_{0}^{\infty} e^{(x-1+p) t} f(x) d x
$$

where $f(x)$ is the density function of $X$ and $p \in \mathbb{R}$.

From (10) and (68), we can derive the following equation:

$$
\begin{aligned}
E\left[e^{(X-1+p) t}\right] & =\int_{0}^{\infty} e^{(x-1+p) t} e^{-x} d x=e^{-t+p t} \cdot \int_{0}^{\infty} e^{-x(1-t)} d x \\
& =\frac{e^{-t}}{1-t} e^{p t}=\sum_{n=0}^{\infty} D_{n}(p) \frac{t^{n}}{n !} .
\end{aligned}
$$

On the other hand, by Taylor expansion, we get

$$
E\left[e^{(X-1+p) t}\right]=\sum_{n=0}^{\infty} E\left[(X-1+p)^{n}\right] \frac{t^{n}}{n !} .
$$

Therefore, by (69) and (70), we obtain the following theorem.
Theorem 17. For $n \geq 0, X \sim \Gamma(1,1)$, the moment of $X-1+p$ is given by

$$
E\left[(X-1+p)^{n}\right]=D_{n}(p)
$$

When $p=0, D_{n}=D_{n}(0)=E\left[(X-1)^{n}\right],(n \geq 0)$.

Thus, we note that

$$
D_{n}=\sum_{l=0}^{n}\left(\begin{array}{l}
n \\
l
\end{array}\right)(-1)^{n-l} E\left[X^{l}\right]
$$

For $X \sim \Gamma(1,1)$, we note that the moment of $X$ is given by $E\left[X^{n}\right]=n !,(n \geq 0)$.

Therefore, by (72), we obtain the following corollary.

Corollary 18. For $n \geq 0, X \sim \Gamma(1,1)$, we have

$$
\begin{gathered}
D_{n}=\sum_{l=0}^{n}\left(\begin{array}{l}
n \\
l
\end{array}\right)(-1)^{n-l} l !, \\
D_{n}(p)=\sum_{l=0}^{n}\left(\begin{array}{l}
n \\
l
\end{array}\right)(p-1)^{n-l} l ! .
\end{gathered}
$$

For $X \sim \Gamma(1,1)$, we have

$$
E\left[e^{(X-1+p+i q) t}\right]=\frac{e^{-t}}{1-t} e^{(p+i q) t}
$$

where $p, q \in \mathbb{R}$.

From (74), we note that

$$
E\left[e^{(X-1+p-i q) t}\right]=\frac{e^{-t}}{1-t} e^{(p-i q) t}
$$

By (74) and (75), we get

$$
\begin{aligned}
E\left[e^{(X-1+p+i q) t}\right]+E\left[e^{(X-1+p-i q) t}\right] & =\frac{2 e^{-t}}{1-t} e^{p t} \cos q t \\
& =\sum_{n=0}^{\infty} 2 D_{n}^{(c)}(p, q) \frac{t^{n}}{n !} .
\end{aligned}
$$

On the other hand, by Taylor expansion, we get

$$
\begin{aligned}
E\left[e^{(X-1+p+i q) t}\right]+E\left[e^{(X-1+p-i q) t}\right]= & \sum_{n=0}^{\infty} E\left[(X-1+p+i q)^{n}\right. \\
& \left.+(X-1+p-i q)^{n}\right] \frac{t^{n}}{n !}
\end{aligned}
$$

Therefore, by (76) and (77), we obtain the following theorem. 
Theorem 19. For $n \geq 0, X \sim \Gamma(1,1)$, we have

$$
E\left[\frac{(X-1+p+i q)^{n}+(X-1+p-i q)^{n}}{2}\right]=D_{n}^{(c)}(p, q) \text {. }
$$

It is easy to show that

$$
\begin{aligned}
E\left[e^{(X-1+p+i q) t}\right]-E\left[e^{(X-1+p-i q) t}\right] & =2 i \frac{e^{-t}}{1-t} e^{p t} \sin q t \\
& =(2 i) \sum_{n=1}^{\infty} D_{n}^{(s)}(p, q) \frac{t^{n}}{n !},
\end{aligned}
$$

where $X \sim \Gamma(1,1)$.

Thus, we have

$$
E\left[\frac{(X-1+p+i q)^{n}-(X-1+p-i q)^{n}}{2 i}\right]=D_{n}^{(s)}(p, q),(n \geq 0),
$$

where $X \sim \Gamma(1,1)$.

\section{Conclusion}

The introduction of derangement numbers $D_{n}$ goes back to as early as 1708 when Pierre Rémond de Montmort considered some counting problem on derangements. In this paper, we dealt with derangement polynomials $D_{n}(x)$ which are natural extensions of the derangement numbers. We showed a recurrence relation for derangement polynomials. We derived identities involving derangement polynomials, Bell polynomials, and Stirling numbers of both kinds. In addition, we also obtained an identity relating Bell polynomials, derangement polynomials, and Euler numbers. Next, we introduced the cosine-derangement polynomials $D_{n}^{(c)}(x, y)$ and sine-derangement polynomials $D_{n}^{(s)}(x, y)$, by means of derangement polynomials. Then, we derived, among other things, their explicit expressions and recurrence relations. Lastly, as applications, we showed that if $X$ is the gamma random variable with parameters 1,1 , then $D_{n}(p), D_{n}^{(c)}(p, q)$, $D_{n}^{(s)}(p, q)$ are given by the "moments" of some variants of $X$.

We have witnessed that the study of some special numbers and polynomials was done intensively by using several different means, which include generating functions, combinatorial methods, umbral calculus, $p$-adic analysis, probability theory, special functions, and differential equations. Moreover, the same has been done for various degenerate versions of quite a few special numbers and polynomials in recent years with their interests not only in combinatorial and arithmetical properties but also in their applications to symmetric identities, differential equations, and probability theories. It would have been nicer if we were able to find abundant applications in other disciplines.

It is one of our future projects to continue to investigate many ordinary and degenerate special numbers and polynomials by various means and find their applications in physics, science, engineering, and mathematics.

\section{Data Availability}

No data were used to support this study.

\section{Conflicts of Interest}

The authors declare that there are no conflicts of interest regarding the publication of this paper.

\section{References}

[1] L. Carlitz, "The number of derangements of a sequence with given specification," The Fibonacci Quarterly, vol. 16, no. 3, pp. 255-258, 1978.

[2] R. J. Clarke and M. Sved, "Derangements and Bell numbers," Mathematics Magazine, vol. 66, no. 5, pp. 299-303, 2018.

[3] T. Kim and D. S. Kim, "Some identities on derangement and degenerate derangement polynomials," in Advances in Mathematical Inequalities and Applications, pp. 265-277, Trends Math., Birkhäuser/Springer, Singapore, 2018.

[4] T. Kim, D. S. Kim, D. V. Dolgy, and J. Kwon, "Some identities of derangement numbers," Proceedings of the Jangjeon Mathematical Society, vol. 21, no. 1, pp. 125-141, 2018.

[5] T. Kim, D. S. Kim, G.-W. Jang, and J. Kwon, "A note on some identities of derangement polynomials," Journal of Inequalities and Applications, vol. 2018, no. 1, 2018.

[6] L. Pro Comtet, Advanced Combinatorics, the Art of Finite and Infinite Expansions, D. Reidel Publishing Co., Dordrecht, 1974.

[7] J.-L. Lavoie and R. Tremblay, "A note on the number of derangements," Jnanabha, vol. 9, no. 10, pp. 139-142, 1980.

[8] I. P. Goulden and D. M. Jackson, "A correspondence between plane planted chromatic trees and generalised derangements," The Bulletin of the London Mathematical Society, vol. 13, no. 1, pp. 28-32, 1981.

[9] D. M. Jackson, "Laguerre polynomials and derangements," Mathematical Proceedings of the Cambridge Philosophical Society, vol. 80, no. 2, pp. 213-214, 1976.

[10] J. B. Remmel, "A note on a recursion for the number of derangements," European Journal of Combinatorics, vol. 4, no. 4, pp. 371-374, 1983.

[11] S. Roman, "The umbral calculus," in Pure and Applied Mathematics, p. 111, Academic Press, Inc. [Harcourt Brace Jovanovich, Publishers], New York, 1984.

[12] T. Kim, Y. Yao, D. S. Kim, and H.-I. Kwon, "Some identities involving special numbers and moments of random variables," The Rocky Mountain Journal of Mathematics, vol. 49, no. 2, pp. 521-538, 2019.

[13] M. D. Springer and W. E. Thompson, "The distribution of products of beta, gamma and Gaussian random variables," SIAM Journal on Applied Mathematics, vol. 18, no. 4, pp. 721-737, 1970.

[14] S. M. Ross, Introduction to Probability Models, Twelfth Edition of [MR0328973], Academic Press, London, 2019.

[15] J. Brillhart, "Note on the single variable Bell polynomials," The American Mathematical Monthly, vol. 74, no. 6, pp. 695-696, 1967.

[16] D. S. Kim, T. Kim, and H. Lee, "A note on degenerate Euler and Bernoulli polynomials of complex variable," Symmetry, vol. 11, no. 9, p. 1168, 2019. 
[17] Y. Simsek, "Identities on the Changhee numbers and Apostoltype Daehee polynomials," Advanced Studies in Contemporary Mathematics (Kyungshang), vol. 27, no. 2, pp. 199-212, 2017.

[18] W. K. Jeong, "Some identities for degenerate cosine(sine)Euler polynomials," Advanced Studies in Contemporary Mathematics (Kyungshang), vol. 30, no. 1, pp. 155-164, 2020.

[19] D. G. Zill and M. R. Cullen, Advanced Engineering Mathematics, Jones and Barrtlett, Mississauga, ON, Canada, 3rd edition, 2006.

[20] B. Simsek, "Some identities and formulas derived from analysis of distribution functions including Bernoulli polynomials and Stirling numbers," Filomat, vol. 34, no. 2, pp. 521-527, 2020.

[21] B. Simsek, "Formulas derived from moment generating functions and Bernstein polynomials," Applicable Analysis and Discrete Mathematics, vol. 13, no. 3, pp. 839-848, 2019.

[22] B. Simsek and B. Simsek, "The computation of expected values and moments of special polynomials via characteristic and generating functions," AIP Conference Proceedings, vol. 1863, pp. 300012-1300012-5, 2017. 